\title{
Subarachnoid Hemorrhage Due to Rupture of an Intracavernous Carotid Artery Aneurysm Coexisting with a Prolactinoma under Cabergoline Treatment
}

\author{
Nobuyuki Akutsu ${ }^{1}$ Kohkichi Hosoda ${ }^{1}$ Kohei Ohta ${ }^{1}$ Hirotomo Tanaka ${ }^{1}$ Masaaki Taniguchi ${ }^{1}$ \\ Eiji Kohmura ${ }^{1}$ \\ ${ }^{1}$ Department of Neurosurgery, Kobe University Graduate School of \\ Medicine, Hyogo, Japan \\ J Neurol Surg Rep 2014;75:e73-e76.

\begin{abstract}
Address for correspondence Nobuyuki Akutsu, MD, Department of Neurosurgery, Kobe University Graduate School of Medicine, 7-5-1 Kusunoki-cho, Chuo-ku, Kobe 650-0017, Japan
\end{abstract} \\ (e-mail: nakutsu@med.kobe-u.ac.jp).
}

\begin{abstract}
We report an unusual case of subarachnoid hemorrhage caused by intraoperative rupture of an intracavernous carotid artery aneurysm coexisting with a prolactinoma. A 58-year-old man presenting with diplopia was found to have a left intracavernous carotid artery aneurysm encased by a suprasellar tumor on magnetic resonance imaging. His serum prolactin level was $5036 \mathrm{ng} / \mathrm{mL}$. Proximal ligation of the left internal carotid artery with a superficial temporal artery to middle cerebral artery anastomosis was scheduled. Because the patient's diplopia had deteriorated, we started him on cabergoline at a dose of $0.25 \mathrm{mg}$ once a week. One month after administration of cabergoline,

Keywords

- intracavernous aneurysm

- subarachnoid hemorrhage

- prolactinoma

- cabergoline the diplopia was improved to some extent and serum prolactin was decreased to 290 $\mathrm{ng} / \mathrm{ml}$. Six weeks after starting the cabergoline, the patient underwent a left frontotemporal craniotomy to treat the aneurysm. When the dura mater was opened, abnormal brain swelling and obvious subarachnoid hemorrhage were observed. Postoperative computed tomography demonstrated a thick subarachnoid hemorrhage. This case suggests that medical therapy for a pituitary adenoma should be started after treatment for a coexisting intracavernous aneurysm is completed.
\end{abstract}

\section{Introduction}

The presence of an intracranial aneurysm coexisting with a pituitary adenoma is well documented. ${ }^{1,2}$ Although several cases of unruptured aneurysms coexisting with pituitary adenomas have been reported, subarachnoid hemorrhage (SAH) caused by rupture of a coexisting intracavernous aneurysm is rare. We report an unusual case of SAH caused by intraoperative rupture of an intracavernous carotid artery aneurysm coexisting with a prolactinoma under cabergoline treatment.

\section{Case Report}

A 58-year-old man presented with diplopia. Neurologic examination revealed left abducens nerve palsy. The patient's visual acuity and visual field were normal. Magnetic resonance imaging (MRI) showed a pituitary tumor with suprasellar extension, associated with a small intratumoral hemorrhage. An enlarged flow void was also noted in the left cavernous sinus, suggesting the presence of an aneurysm at the intracavernous portion of the internal carotid artery (ICA) (-Fig. 1A, B). We concluded that the intratumoral hemorrhage was apoplexy because the hemorrhage was remote to the aneurysm. Cerebral angiography revealed a large intracavernous fusiform aneurysm of the left ICA ( - Fig. 1C, D). The patient tolerated a balloon test occlusion (BTO) of the left ICA well, and angiography showed good collateral blood supply through the anterior communicating artery. However, the mean stump pressure during BTO was received

May 2, 2013

accepted after revision

November 16, 2013

published online

March 12, 2014
DOI http://dx.doi.org/

10.1055/s-0033-1364166. ISSN 2193-6358.
๑) 2014 Georg Thieme Verlag KG,
Stuttgart · New York

License terms

((1) $\circledast$ 


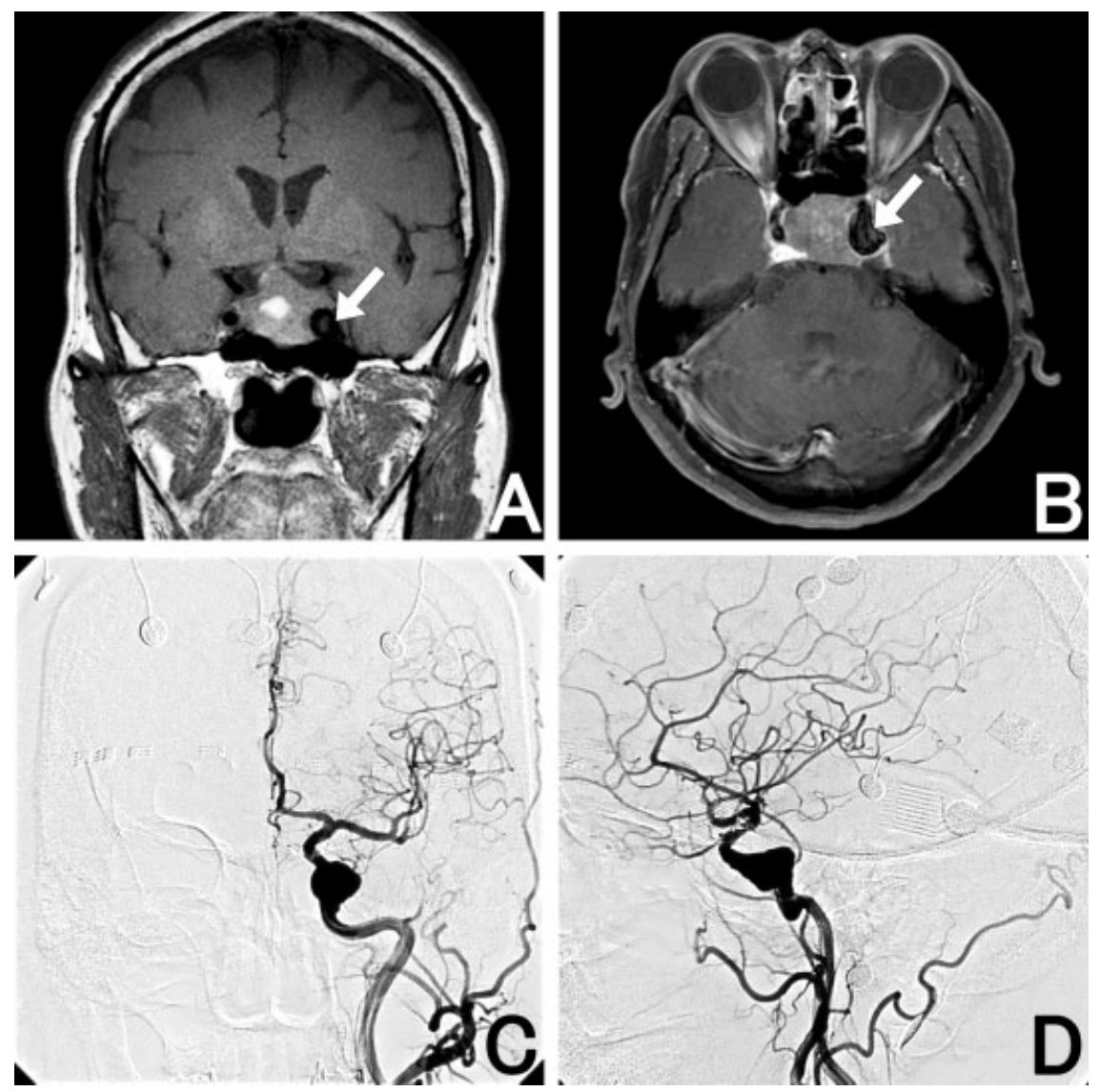

Fig. 1 (A, B) Magnetic resonance imaging (MRI) shows an intra- and suprasellar tumor associated with intratumoral hemorrhage and an enlarged flow void (arrow) in the left cavernous sinus, suggesting an aneurysm. (C, D) Left carotid artery angiography reveals a large intracavernous fusiform aneurysm of the left internal carotid artery. (A) Coronal T1-weighted MRI. (B) Gadolinium-enhanced axial T1-weighted MRI. (C) Left carotid artery angiography (anterior-posterior view). (D) Left carotid artery angiography (lateral view).

$45 \mathrm{~mm} \mathrm{Hg},<50 \%$ of the preocclusion mean arterial pressure, which indicated the need for a low-flow bypass graft according to hospital policy. Proximal ligation of the left ICA with a superficial temporal artery to the middle cerebral artery (STA-MCA) anastomosis was scheduled. Hormonal assessment revealed an elevated serum prolactin level of 5036 $\mathrm{ng} / \mathrm{mL}$ (normal: 3.6-12.8 ng/mL). We considered that both the prolactinoma and aneurysm were responsible for the abducens palsy. Because the patient's diplopia had deteriorated, we started him on cabergoline at a dose of $0.25 \mathrm{mg}$ once a week. One month after administration of cabergoline, the diplopia was improved to some extent; serum prolactin was decreased to $290 \mathrm{ng} / \mathrm{ml}$.

Six weeks after starting the cabergoline, the patient underwent a left frontotemporal craniotomy to treat the aneurysm. When the dura mater was opened, the brain swelled and an SAH was clearly observed. It was essentially impossible to continue the operation, so only a decompressive craniectomy was performed. Computed tomography immediately after the surgery revealed a thick SAH (-Fig. 2). The patient was managed by intubation and sedated with propofol for 14 days. When sedation was stopped, the patient awakened with mild sensory aphasia and dysphasia.

A second operation was performed 6weeks after the first. Proximal ligation of the left ICA with an STA-MCA anastomo- sis and cranioplasty were completed uneventfully. No new neurologic deficits developed after the procedure. Postoperative angiography revealed disappearance of the aneurysm and patency of the STA-MCA anastomosis (-Fig. 3A). MRI confirmed tumor regression and disappearance of the left intracavernous flow void (-Fig. 3B).

\section{Discussion}

The differential diagnosis for SAH in the present case could be divided into two categories: aneurysmal rupture and pituitary apoplexy. There is no pathologic evidence of what caused the hemorrhage in our case. Although SAH is one of the presentations of pituitary apoplexy, broad and massive SAH is rare. ${ }^{3}$ However, visual deterioration is the cardinal sign of pituitary apoplexy and was reported to occur in $80 \%$ of 38 cases of major pituitary apoplexy. ${ }^{4}$ The present case did not present with visual deterioration after the presentation of $\mathrm{SAH}$, and postoperative MRI demonstrated no remnants of intratumoral hemorrhage. Thus we conclude that a ruptured aneurysm was the cause of SAH in the present case.

There have been very few reports of SAH caused by rupture of intracavernous aneurysms coexisting with pituitary adenomas. Only two cases of pituitary apoplexy caused by aneurysmal bleeding into pituitary adenomas have been 

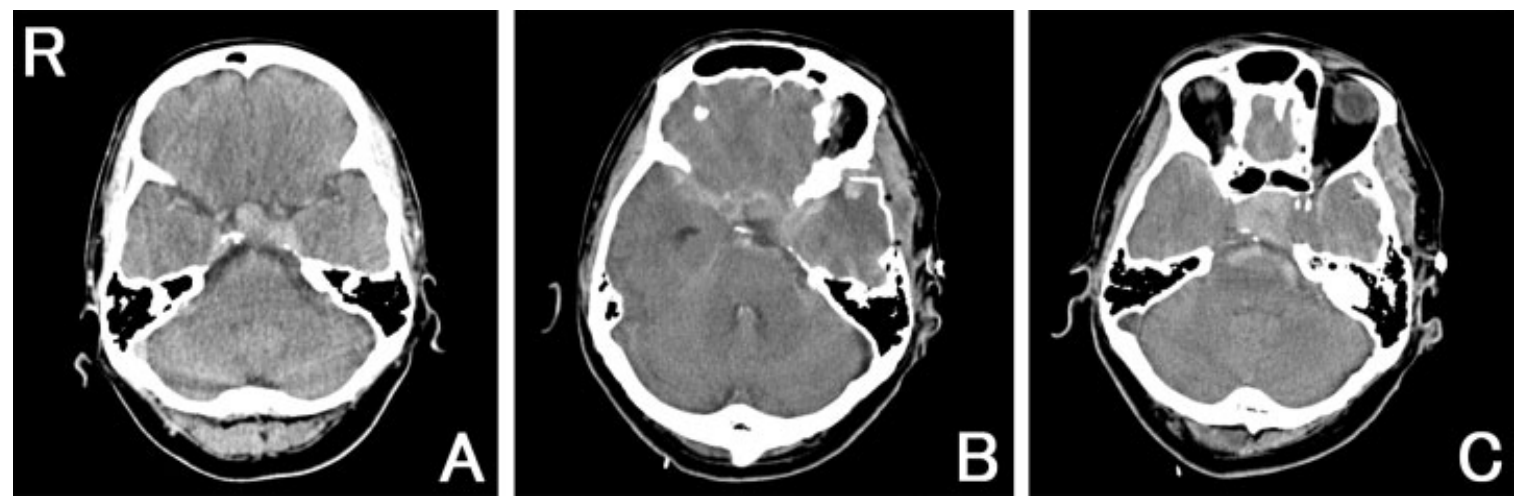

Fig. 2 (A) Preoperative computed tomography (CT) scan shows a pituitary tumor with suprasellar extension. (B, C) Postoperative CT scans immediately after the first surgery show tumor regression and a thick diffuse subarachnoid hemorrhage (SAH) continuing to the intrasellar space.

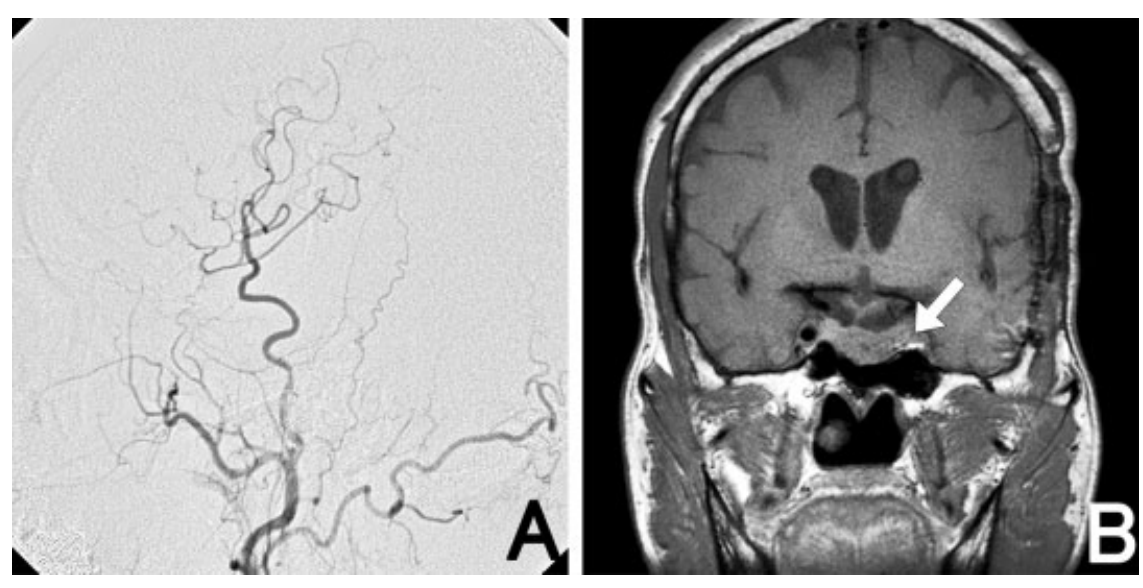

Fig. 3 Postoperative angiography (left) shows good patency of the superficial temporal artery graft to the middle cerebral artery. Gadoliniumenhanced coronal magnetic resonance imaging after the second operation (right) reveals a thrombosed aneurysm (arrow) and tumor regression compared with before cabergoline treatment.

reported. ${ }^{5,6}$ Suzuki et al suggested that the tumor in their case might have invaded and weakened the aneurysmal wall, causing the aneurysm to grow and subsequently rupture into the tumor. ${ }^{6}$ Imamura et al reported a case of fatal epistaxis caused by rupture of an intracavernous aneurysm coexisting with a pituitary adenoma before surgery. ${ }^{7}$

The effect of adenoma shrinkage as a result of medical therapy on a coexisting intracavernous aneurysm has been unclear. Hori et al reported the enlargement of an aneurysm contiguous with a growth hormone-producing pituitary adenoma as the result of tumor regression by bromocriptine therapy, ${ }^{8}$ and they suggested that the traction force, which was produced by the regression, contributed to the enlargement. In our case, we speculate that an aperture between the adenoma and intracavernous aneurysm was created and widened by shrinkage of the prolactinoma by cabergoline. The surgical stress and acute decompression from the craniotomy caused the aneurysm to rupture, resulting in SAH.

\section{Conclusion}

In a case of an intracavernous aneurysm coexisting with a functional pituitary macroadenoma, medical therapy for the pituitary adenoma should be started only after treatment for the coexisting intracavernous aneurysm has been completed, to avoid any life-threatening hemorrhagic complications. However, in the case of acute visual deterioration as a consequence of the intratumoral hemorrhage, cabergoline administration of a small amount may be acceptable to prevent the deterioration of the symptom.

\section{References}

1 Acqui M, Ferrante L, Fraioli B, Cosentino F, Fortuna A, Mastronardi L. Association between intracranial aneurysms and pituitary adenomas. AEtiopathogenetic hypotheses. Neurochirurgia (Stuttg) 1987; 30(6):177-181

2 Pant B, Arita K, Kurisu K, Tominaga A, Eguchi K, Uozumi T. Incidence of intracranial aneurysm associated with pituitary adenoma. Neurosurg Rev 1997;20(1):13-17

3 Nakahara K, Oka H, Utsuki S, et al. Pituitary apoplexy manifesting as diffuse subarachnoid hemorrhage. Neurol Med Chir (Tokyo) 2006;46(12):594-597

4 Wakai S, Fukushima T, Teramoto A, Sano K. Pituitary apoplexy: its incidence and clinical significance. J Neurosurg 1981;55(2):187-193

5 Chuang CC, Chen YL, Pai PC. A giant intracavernous carotid artery aneurysm embedded in pituitary macroadenoma presenting with pituitary apoplexy. Cerebrovasc Dis 2006;21(1-2):142-144 
e76 Ruptured Aneurysm Coexisting with a Prolactinoma Akutsu et al.

6 Suzuki H, Muramatsu M, Murao K, Kawaguchi K, Shimizu T. Pituitary apoplexy caused by ruptured internal carotid artery aneurysm. Stroke 2001;32(2):567-569

7 Imamura J, Okuzono T, Okuzono Y. Fatal epistaxis caused by rupture of an intratumoral aneurysm enclosed by a large prolac- tinoma-case report. Neurol Med Chir (Tokyo) 1998;38(10): 654-656

8 Hori T, Muraoka K, Hokama Y, Takami M, Saito Y. A growthhormone-producing pituitary adenoma and an internal carotid artery aneurysm. Surg Neurol 1982;18(2):108-111 